\title{
Solvable Dynamics in a System of Interacting Random Tops
}

\author{
Felix Ritort* \\ Institute for Theoretical Physics, University of Amsterdam, Valckenierstraat 65, 1018 XE Amsterdam, The Netherlands
}

(Received 21 May 1997)

\begin{abstract}
A new solvable model of synchronization dynamics is introduced. It consists of a system of long range interacting tops or magnetic moments with random precession frequencies. The model allows for an explicit study of orientational effects in synchronization phenomena as well as nonlinear processes in resonance phenomena in strongly coupled magnetic systems. A stability analysis of the incoherent solution is performed for different types of orientational disorder. A system with orientational disorder always synchronizes in the absence of noise. [S0031-9007(97)04832-1]
\end{abstract}

PACS numbers: 05.40. $+\mathrm{j}$, 05.70.Fh, 87.10.+e

Much theoretical effort has been devoted to the study of synchronization dynamics in simple systems composed of interacting units. In those cases there is competition between oscillations arising from the natural randomness in the members of a population and macroscopic synchronization of the population as a whole. It is widely believed that these models provide a plausible explanation for the existence of synchronization phenomena in a large variety of physical systems ranging from physics to biology [1]

A simple model which describes the emergence of synchronization phenomena in a population of phase oscillators was proposed many years ago by Kuramoto [2]. Despite extensive studies in the past, there are still some open issues such as a the study of the dynamics in the absence of external noise [3] or the onset of synchronization in the critical region [4,5]. Only very recently, a physical realization of the Kuramoto model has been found [6].

The aim of this Letter is to introduce a new model which shows a new mechanism for synchronization phenomena. The fundamental new feature of this model is that it explicitly introduces the role of orientational degrees of freedom in the synchronization dynamics. This feature is ubiquitous in nature in systems formed by units with natural magnetic (or angular) moment. Hence the proposed model in this Letter is a step towards a microscopic semiclassical theory of nonlinear phenomena in magnetic resonance processes (and, in particular, ferromagnetic resonance [7]) as well as synchronization phenomena in biomagnetism (and, in particular, responses of living organisms to external magnetic fields).

The model consists of a system of $N$ tops (or magnetic moments), each one characterized by a random natural precession vector $\vec{\omega}_{i}$, interacting ferromagnetically in a meanfield way. Randomness in magnetic systems can arise due to local inhomogeneities or crystal field anisotropies. The tops are specified by a three component unit vector $\vec{x}_{i}$ $(i=1, N)$ and obey the following dynamics:

$$
\frac{\partial \vec{x}_{i}}{\partial t}=-\frac{\partial \mathcal{H}}{\partial \vec{x}_{i}}+\vec{\omega}_{i} \times \vec{x}_{i}+\vec{\eta}_{i}
$$

where the $\vec{\omega}_{i}$ are random quenched precession vectors, $\mathcal{H}$ is the Hamiltonian of the system, and $\vec{\eta}_{i}$ is an external white noise with zero mean and correlation $\left\langle\vec{\eta}_{i}(t) \vec{\eta}_{j}\left(t^{\prime}\right)\right\rangle=$ $6 T \delta_{i j} \delta\left(t-t^{\prime}\right)$. The Hamiltonian $\mathcal{H}$ is given by

$$
\mathcal{H}=-\frac{K}{N} \sum_{i<j} \vec{x}_{i} \vec{x}_{j}-\sum_{i} m_{i} \vec{x}_{i}^{2},
$$

where the $m_{i}$ are Lagrange multipliers introduced in order to ensure the local constraint $\left|\vec{x}_{i}\right|=1$ at all times. Note that in the dynamics there is competition between two opposite effects: a ferromagnetic interaction, which tries to align the tops in the same direction, and a natural precession of the tops around random quenched directions, which drives the system to an incoherent state. The competition between a dissipative force $\left(-\frac{\partial \mathcal{H}}{\partial \vec{x}_{i}}\right)$ and a driving force $\left(\vec{\omega}_{i} \times \vec{x}_{i}\right)$ which pumps energy into the system is essential for the emergence of nonlinear oscillations and synchronization dynamics in the model. In resonance experiments this last term mimics some external resonance magnetic field which induces Larmor precession in the magnetic moments.

To analyze the previous dynamics it is convenient to introduce polar coordinates for the tops $\vec{x}_{i}=\left(\sin \theta_{i} \cos \phi_{i}, \sin \theta_{i} \sin \phi_{i}, \cos \theta_{i}\right)$ as well as for the random precessions $\vec{\omega}_{i}=\omega_{i}\left(\sin \mu_{i} \cos \lambda_{i}, \sin \mu_{i} \sin \lambda_{i}\right.$, $\left.\cos \mu_{i}\right)$. It is not difficult to check that dynamical equation (1) can be written in the following way:

$$
\begin{gathered}
\frac{\partial \theta_{i}}{\partial t}=-K\left(C \sin \theta_{i}+A \cos \theta_{i} \cos \phi_{i}+B \cos \theta_{i} \sin \phi_{i}\right) \\
+\omega_{i} \sin \mu_{i} \sin \left(\lambda_{i}-\phi_{i}\right)+T \cot \theta_{i}+\alpha_{i} \\
\frac{\partial \phi_{i}}{\partial t} \sin \theta_{i}=-K\left(A \sin \phi_{i}-B \cos \phi_{i}\right) \\
+\omega_{i}\left[-\sin \mu_{i} \cos \theta_{i} \cos \left(\lambda_{i}-\phi_{i}\right)\right. \\
\left.+\cos \mu_{i} \sin \theta_{i}\right]+\beta_{i},
\end{gathered}
$$

where $\quad N A=\sum_{j} \cos \phi_{j} \sin \theta_{j}, N B=\sum_{j} \sin \phi_{j} \sin \theta_{j}$, $N C=\sum_{j} \cos \theta_{j}$, and the $\alpha_{i}, \beta_{i}$ are white noises with variance $2 T$.

Solving the previous dynamical equations seems at first glance a rather difficult task. Here we will follow a powerful approach recently introduced for the study of 
the Kuramoto model [8] by considering an appropriate set of moments which is invariant under the symmetry of the original dynamical equations (1). Before studying the most general case, and for sake of simplicity, we will first consider the case where there are no frequencies, i.e., $\omega_{i}=0$.

The non-disordered case $\omega_{i}=0 .-\mathrm{It}$ is easy to observe from Eq. (1) that dynamics of the model is invariant under the group of spatial rotations whose generators are $L^{2}$ and $L^{z}$ and the eigenfunctions are the spherical harmonics. The most natural set of moments which can close the dynamics is

$$
M_{l m}(t)=\frac{1}{N} \sum_{i=1}^{N} Y_{l m}\left[\theta_{i}(t), \phi_{i}(t)\right],
$$

where the $Y_{l m}(\theta, \phi)=C_{l m} P_{l}^{m}(\theta) \exp (i m \phi)$ are the spherical harmonics with $C_{l m}=[(2 l+1)(l-m) ! /$ $4 \pi(l+m) !]^{1 / 2}$ as normalization constants. $P_{l}^{m}$ are the associated Legendre polynomials where $-l \leq m \leq l$.

It is not difficult to write the equation of motion for the $M_{l m}(t)$. Using the definition of spherical harmonics, simple recursion formulas for the Legendre polynomials as well as the Gaussian representation of the noise, it is possible to show that the moments obey the following closure equation:

$$
\begin{aligned}
\frac{\partial M_{l, m}}{\partial t}=K[ & C\left(a_{l, m} M_{l-1, m}-b_{l, m} M_{l+1, m}\right) \\
& -(A-i B)\left(c_{l, m} M_{l-1, m+1}+d_{l, m} M_{l+1, m+1}\right) \\
& \left.+(A+i B)\left(e_{l, m} M_{l-1, m-1}+f_{l, m} M_{l+1, m-1}\right)\right] \\
- & T l(l+1) M_{l, m},
\end{aligned}
$$

where $a, b, c, d, e$, and $f$ are Clebsch-Gordan-like coefficients given by

$$
\begin{gathered}
a_{l, m}=(l+1) \sqrt{\frac{l^{2}-m^{2}}{4 l^{2}-1}} ; \\
b_{l, m}=l \sqrt{\frac{(l+1)^{2}-m^{2}}{(2 l+1)(2 l+3)}}, \\
c_{l, m}=\frac{(l+1)}{2} \sqrt{\frac{(l-m)(l-m-1)}{4 l^{2}-1}} ; \\
d_{l, m}=\frac{l}{2} \sqrt{\frac{(l+m+1)(l+m+2)}{(2 l+1)(2 l+3)}},
\end{gathered}
$$

and $e_{l, m}=c_{l,-m}, f_{l, m}=d_{l,-m}$. The time dependent parameters $A, B, C$ in (6) are given by $A+i B=\sqrt{8 \pi / 3} M_{1,1}, C=\sqrt{4 \pi / 3} M_{1,0}$. Note that the dynamical equation (6) is invariant under the transformation $m \rightarrow-m$ if the moments satisfy the relation $M_{l,-m}=(-1)^{m} M_{l, m}^{*}$, which is indeed the relation satisfied by the spherical harmonics $Y_{l, m}$. The recursion relations Eq. (6) show explicitly that the dynamics has been closed. The special case $K=0$ corresponds to the random walk on a spherical surface, a case well known in the literature [9]. Then Eq. (6) trivially reduces to $\dot{M}_{l, m}=-T l(l+1) M_{l, m}$, which shows that all moments decay exponentially fast to zero (except $M_{0,0}$, which is a constant of motion equal to $1 / \sqrt{4 \pi}$ ). Equation (6) can be expressed in a more intuitive form introducing an appropriate generating function. To see this, let us define

$$
\begin{aligned}
g_{t}(\theta, \phi) & =\sum_{l=0}^{\infty} \sum_{m=-l}^{l} M_{l, m}(t) Y_{l, m}(\theta, \phi) \\
& =\frac{1}{N} \sum_{j=1}^{N} \delta\left(\cos \theta_{j}-\cos \theta\right) \delta\left(\phi_{j}-\phi\right),
\end{aligned}
$$

which is nothing else than the probability density to find a top with a given solid angle $\Omega=(\theta, \phi)$ on the unit sphere. The last equality in Eq. (9) comes from the closure condition of the spherical harmonics. Using Eq. (6) it is not difficult to derive the following equation for $g_{t}: \frac{\partial g_{t}}{\partial t}=-\operatorname{div}\left[g_{t} \vec{v}(\theta, \phi)\right]+T \nabla^{2} g_{t}$, where $\vec{v}=\left(v_{\theta}, v_{\phi}\right)$ is a two component velocity field given by $v_{\theta}=K r[\sin \Theta \cos \theta \cos (\Phi-\phi)-\sin \theta \cos \Theta] ; v_{\phi}=$ $K r \sin \Theta \sin (\Phi-\phi)$.

The time dependent parameters $r, \Theta$, and $\Phi$ which appear in the velocity field are self-consistently computed from the probability distribution $g_{t}$. They are given by $A=r \sin (\Theta) \cos (\Phi) ; B=r \sin (\Theta) \sin (\Phi) ; C=\cos (\Theta)$, where $A, B$, and $C$ have been introduced before. $r=$ $\sqrt{A^{2}+B^{2}+C^{2}}$ is the synchronization parameter (i.e., the length of the global magnetization) and measures the degree of coherence of the tops. The previous equation seems hardly manageable but still some results can be inferred, in particular, the nature of the stationary solutions. It is easy to check that these are solutions of the Boltzmann type, i.e., equilibrium solutions of the Hamiltonian Eq. (1). This is expected since the model, in the absence of random precessions, is purely relaxational.

The disordered case $\omega_{i} \neq 0$. - In the presence of random precessions the model is not purely relaxational since there are external driving random forces. In this case we expect the emergence of a rich dynamical behavior due to the competition between the ordering ferromagnetic interaction and the random natural precessions of the tops. For the sake of simplicity and in order to investigate the effect of orientational disorder, we will consider here the case in which the precession angular velocities have the same magnitude (i.e., $\omega_{i}=\omega$ ) but point in different directions in space. In this case the disorder is specified by a probability distribution $p(\mu, \lambda)$. It is easy to generalize the definition of the moments (5) to include the presence of quenched disorder. Now the moments are characterized by four quantum numbers [10], two of them appearing as a consequence of the disorder,

$$
M_{l, m}^{p, q}=\frac{1}{N} \sum_{i=1}^{N} Y_{l, m}\left(\theta_{i}, \phi_{i}\right) Y_{p, q}\left(\mu_{i}, \lambda_{i}\right) .
$$

After some algebra, the closure equations for the new set of moments read 


$$
\begin{aligned}
\frac{\partial M_{l, m}^{p, q}}{\partial t}= & K\left[C\left(a_{l m} M_{l-1, m}^{p, q}-b_{l, m} M_{l+1, m}^{p, q}\right)-(A-i B)\left(c_{l, m} M_{l-1, m+1}^{p, q}+d_{l, m} M_{l+1, m+1}^{p, q}\right)\right. \\
& \left.+(A+i B)\left(e_{l, m} M_{l-1, m-1}^{p, q}+f_{l, m} M_{l+1, m-1}^{p, q}\right)\right] \\
- & T l(l+1) M_{l, m}^{p, q}+\frac{i \omega}{2}\left(A_{l, m}^{p, q} M_{l, m}^{p-1, q+1}-B_{l, m}^{p, q} M_{l, m-1}^{p+1, q+1}-C_{l, m}^{p, q} M_{l, m}^{p-1, q-1}+D_{l, m}^{p, q} M_{l, m}^{p+1, q-1}\right) \\
+ & i m \omega\left(E_{p, q} M_{l, m}^{p+1, q}+F_{p, q} M_{l, m}^{p-1, q}\right),
\end{aligned}
$$

where the expressions for $a, b, c, d, e$, and $f$ have been given in Eqs. (7) and (8) and the other coefficients are

$$
\begin{gathered}
A_{l, m}^{p, q}=\sqrt{\frac{(l-m+1)(l+m)(p-q)(p-q-1)}{4 p^{2}-1} ; \quad E_{p, q}=\sqrt{\frac{(p+q+1)(p-q+1)}{(2 p+1)(2 p+3)}},} \\
B_{l, m}^{p, q}=\sqrt{\frac{(l-m+1)(l+m)(p+q+2)(p+q+1)}{(2 p+1)(2 p+3)}} ; \quad F_{p, q}=\sqrt{\frac{(p-q)(p+q)}{4 p^{2}-1}},
\end{gathered}
$$

and $C_{l, m}^{p, q}=A_{l,-m}^{p,-q}, D_{l, m}^{p, q}=B_{l,-m}^{p,-q}$. The parameters $A, B$, and $C$ have been already defined after Eqs. (3) and (4). In terms of the new set of moments they are given by $A+i B=4 \pi \sqrt{2 / 3} M_{1,1}^{0,0} ; C=4 \pi \sqrt{1 / 3} M_{1,0}^{0,0}$. Note that the moments $M_{0,0}^{p, q}=\frac{1}{\sqrt{4 \pi}} \int d \Omega Y_{p, q}(\Omega) p(\Omega)$ [where $\Omega=(\mu, \lambda)$ is the solid angle and the integration is over all of the unit sphere] are constants of the motion. Let us mention that also in this case a probability distribution, i.e., a generating function for all the moments, can be defined like in the nondisordered case. We will not extend on these considerations and instead we will focus on the novel properties of the model. For this purpose let us consider the case of disorder distributions with axial symmetry around the $z$ axis (the following considerations can be extended easily to the more general case [11]). This means that $p(\Omega)=p(\mu)$. In this case it is possible to study the region in the plane $\tilde{\omega}=\omega / T, \quad \tilde{K}=K / T$, where the incoherent solution $r=0$ is unstable. Our calculation follows essentially the equivalent one for the Kuramoto model $[12,13]$. To this end we expand the moments around the incoherent solution $\quad M_{l, m}^{p, q}=\frac{1}{\sqrt{4 \pi}} \delta_{l, 0} \delta_{m, 0} M_{0,0}^{p, q}+\epsilon \eta_{l, m}^{p, q} \exp (\alpha t)$, where $\epsilon$ is a small parameter. Substituting this result in Eq. (11) and performing a linear stability analysis we find a set of two linear equations (uncoupled from the rest of the modes) involving the two fundamental modes $\eta_{1,0}(\Omega), \eta_{1,1}(\Omega)$. These modes are defined by $\eta_{l, m}^{p, q}=\int d \Omega \eta_{l, m}(\Omega) Y_{p, q}(\Omega)$. After some calculation (details will be shown elsewhere [11]) the condition for the linear stability of the incoherent solution is determined by the roots of the equation $\lambda_{1}(\gamma) \lambda_{2}(\gamma)=0$, where

$$
\begin{array}{r}
\lambda_{1}(\gamma)=\gamma^{3}-\frac{2 \tilde{K}}{3} \gamma^{2}+\tilde{\omega}^{2} \gamma-\frac{2 \tilde{K}}{3} \tilde{\omega}^{2} \overline{\cos ^{2}(\mu)}, \\
\lambda_{2}(\gamma)=\gamma^{3}-\frac{2 \tilde{K}}{3} \gamma^{2}+\tilde{\omega}^{2} \gamma-\frac{\tilde{K}}{3} \tilde{\omega}^{2} \overline{\sin ^{2}(\mu)}, \\
\gamma=\alpha+2,
\end{array}
$$

and $\overline{A(\mu)}=2 \pi \int_{0}^{\pi} \sin (\mu) p(\mu) A(\mu)$. These are two cubic equations and each one yields three roots (one of them is real and the other two are complex conjugates). When the real part of one of these six roots becomes larger than 2 the incoherent solution is unstable. The equation in (14) and (15) that determines the stability is one where the independent $\gamma$ term is the largest (see the examples below). The boundaries of the region where the incoherent solution is unstable indicate the dynamical transition lines. While the stability boundaries will depend on the particular disorder distribution, some general results still can be inferred. In particular, in the absence of noise $T=0$, there is no critical value of $K$ below which the incoherent solution becomes linearly stable. This result is also found in the Kuramoto model with disorder distributions with vanishing probability of oscillators with zero frequency [12], for instance, the bimodal distribution [13]. This last case has been shown to display a very rich dynamical behavior also shared by the present model. Here we are going to consider three cases of disorder distributions which cover a large variety of physical situations: (a) precession vectors lying in the $z$ axis and randomly pointing in opposite directions, i.e., $p(\mu)=0$ except for $\mu=0, \pi$; (b) precession vectors uniformly distributed and lying in the $x-y$ plane, i.e., $p(\mu)=\frac{1}{2 \pi} \delta\left(\mu-\frac{\pi}{2}\right)$; (c) precession vectors isotropically distributed on the sphere, i.e., $p(\mu)=\frac{1}{4 \pi}$.

Case (a) is particularly interesting despite its simplicity. Half of the tops precess in one sense, the other half precess in the other sense, around the $z$ axis. In this case $\cos ^{2}(\mu)=1$ and the stability condition for the incoherent solution is determined by $\lambda_{1}(\gamma)$, which yields $\tilde{K}<3$. For $\tilde{K}>3$ the incoherent solution is always unstable whatever the value of $\tilde{\omega}$. A quick inspection on the definition of the moments Eq. (10) reveals that they reduce to two families $H_{l, m}=M_{l, m}^{0,0}, G_{l, m}=M_{l, m}^{1,0}$. A trivial stationary solution of dynamical equations is then given by $H_{l, m}=H_{l, 0} \delta_{m, 0}, G_{l, m}=0$. This solution coincides with that derived previously in the nondisordered case $\omega_{i}=0$. With an appropriate gauge transformation this case can be shown to be equivalent to the relaxation of a mean-field antiferromagnet [and the stationary solutions in this case are the equilibrium solutions of the Hamiltonian Eq. (2)]. 
Cases (b) and (c) display a more rich dynamical behavior. Case (b) corresponds to all precession directions lying on the $x-y$ plane. In this case $\overline{\sin ^{2}(\mu)}=1$ and the stability criteria is fixed by the roots of $\lambda_{2}(\gamma)$. The incoherent solution is linearly stable for $\tilde{K}<3$ and linearly unstable for $\tilde{K}>6$ and for intermediate values of $\tilde{K}$ depending on the value of $\tilde{\omega}$. More concretely, the incoherent solution is stable for $\tilde{\omega}^{2}>(8 \tilde{K}-$ $24) /(6-\tilde{K})$. Case (c) is the limit situation (i.e., the most orientationally disordered case) with the largest region in the phase diagram where the incoherent solution is linearly stable. In this case, $\cos ^{2}(\mu)=1 / 3$ and $\lambda_{1}(\gamma)=\lambda_{2}(\gamma)$. The incoherent solution is linearly stable for $\tilde{K}<3$, linearly unstable for $\tilde{K}>9$, and stable in the intermediate region $3<\tilde{K}<9$ if $\tilde{\omega}^{2}>(12 \tilde{K}-36) /(9-\tilde{K})$.

The main difference between the present model and the Kuramoto model with bimodal distribution of frequencies lies in their phase diagrams. The neutral stability line for the incoherent solution in the phase diagram $(\tilde{K}, \tilde{\omega})$ has a vertical parabolic branch at $\tilde{K}_{c}: \tilde{\omega} \sim\left(\tilde{K}-\tilde{K}_{c}\right)^{-1 / 2}$ $\left[\tilde{K}_{c}=6,9\right.$ for cases (b) and (c), respectively]. In the Kuramoto model, the neutral stability line is the semirect $\tilde{K}_{c}=4, \tilde{\omega}>1$ [13]. In the region where the incoherent solution is unstable we expect the existence of coherent fixed point solutions as well as oscillatory time dependent solutions. To show this we have numerically solved Eq. (11) for a finite set of moments using a simple second order Euler algorithm. A maximum value of both angular momenta $l$ an $p$ equal to 10 is enough for the calculation (which implies an approximate number of 10000 moments). In Fig. 1 we show the time evolution of the synchronization parameter for model (c) in the

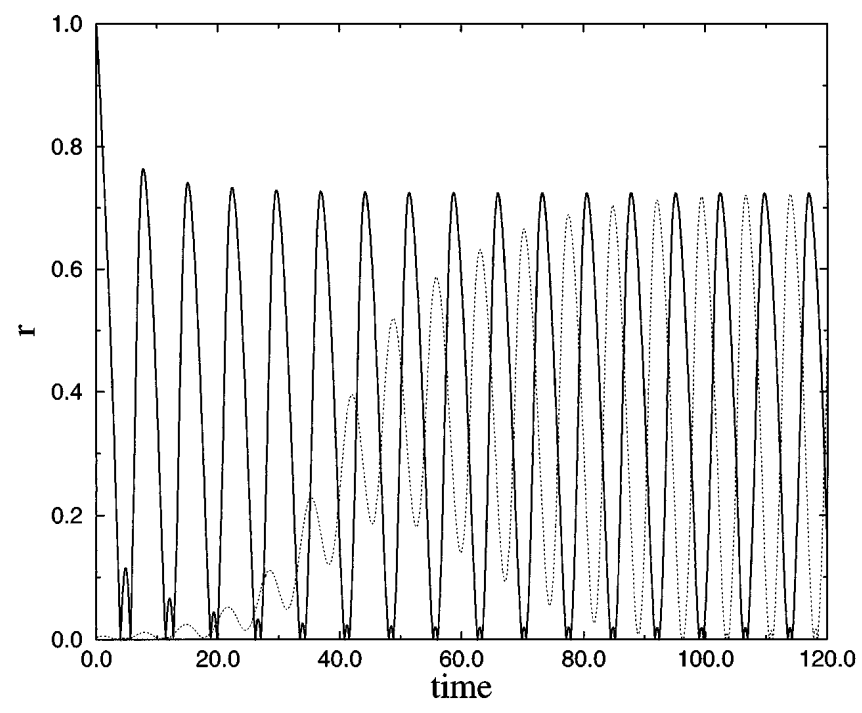

FIG. 1. Synchronization parameter $r$ as a function of time for case (c) at $\tilde{K}=\tilde{\omega}=50 / 3$ starting from two initial conditions: all tops pointing in the same directions (continuous line) and the uncoherent state (dotted line). region where the incoherent solution is unstable starting from two different initial conditions.

To summarize, we have introduced a solvable model of interacting random tops or magnetic moments. The model explicitly introduces orientational effects in the synchronization dynamics, a feature which is not present in the Kuramoto model. These features are extensively found in a large variety of physical systems formed by interacting units with characteristic magnetic moment. In the only presence of orientational disorder we have shown the emergence of synchronized oscillations and nonlinear phenomena (see Fig. 1). These dynamical features are commonly observed in magnetic resonance experiments (spin echoes in NMR, nonlinear processes in ferrites at high frequencies) as well as synchronized responses in living systems in the presence of an external magnetic field. The present model altogether with the Kuramoto model provide simple examples of analytically tractable models with nonrelaxational dynamics. The present study should be extended to include frequency dispersion (i.e., the $\omega_{i}$ different) and external uniform magnetic fields as well as the noise free $(T=0)$ dynamics in this model where the higher modes of the incoherent solution are neutrally stable.

F. R. is grateful to the Foundation for Fundamental Research of Matter (FOM) in The Netherlands for financial support through Contract No. FOM-67596. I acknowledge A. Labarta, I. Pagonabarraga, C. J. Perez-Vicente, and J. M. Rubí for useful discussions and L. L. Bonilla for a careful reading of the manuscript.

*Electronic address: ritort@phys.uva.nl

[1] A.T. Winfree, The Geometry of Biological Time (Springer-Verlag, Berlin, 1980).

[2] Y. Kuramoto, International Symposium on Mathematical Problems in Theoretical Physics. Lectures Notes in Physics (Springer-Verlag, Berlin, 1975), Vol. 39, p. 420.

[3] S. H. Strogatz, R. E. Mirollo, and P.C. Matthews, Phys. Rev. Lett. 68, 2730 (1992).

[4] H. Daido, Phys. Rev. Lett. 73, 760 (1994).

[5] J. D. Crawford, Phys. Rev. Lett. 74, 4341 (1995).

[6] K. Wiesenfeld, P. Colet, and S. H. Strogatz, Phys. Rev. Lett. 76, 404 (1996).

[7] S. V. Vonsovskii, Ferromagnetic Resonance (Pergamon Press, New York, 1966).

[8] C. J. Perez-Vicente and F. Ritort, J. Phys. A (to be published).

[9] W. T. Coffey, Y.P. Kalmykov, and J. T. Waldron, The Langevin Equation (World Scientific, Singapore, 1996).

[10] We will denote the indices of the moments as quantum numbers in analogy with the labeling of eigenstates in quantum mechanics.

[11] F. Ritort (to be published).

[12] S. H. Strogatz and R.E. Mirollo, J. Phys. A 21, 699 (1988); J. Stat. Phys. 63, 613 (1991).

[13] L. L. Bonilla, J. C. Neu, and R. Spigler, J. Stat. Phys. 67, 313 (1992). 